\title{
Determining Chromosomal Arms 1p/19q Co- deletion Status in Low Graded Glioma by Cross Correlation-periodogram Pattern Analysis
}

\section{Debanjali Bhattacharya}

International Institute of Information Technology Bangalore, Networking and Communication

Neelam Sinha ( $\sim$ neelam.sinha@iiitb.ac.in )

International Institute of Information Technology Bangalore, Networking and Communication

Jitender Saini

National Institute of Mental Health and Neurosciences

\section{Research Article}

Keywords: Chromosomal Arms, glioma , diagnosis , surgical biopsy

Posted Date: May 25th, 2021

DOI: https://doi.org/10.21203/rs.3.rs-537094/v1

License: (c) (i) This work is licensed under a Creative Commons Attribution 4.0 International License.

Read Full License

Version of Record: A version of this preprint was published at Scientific Reports on December 1st, 2021. See the published version at https://doi.org/10.1038/s41598-021-03078-1. 


\title{
Determining chromosomal arms $1 \mathrm{p} / 19 q$ co-deletion status in low graded glioma by cross correlation-periodogram pattern analysis
}

\author{
Debanjali Bhattacharya ${ }^{1}$, Neelam Sinha ${ }^{1, *}$, and Jitender Saini ${ }^{2}$ \\ ${ }^{1}$ International Institute of Information Technology Bangalore, Networking and Communication, Bangalore, 560100, \\ India \\ ${ }^{2}$ National Institute of Mental Health and Neuro Science, department of Neurology, Bangalore, 560029, India \\ *neelam.sinha@iiitb.ac.in
}

\begin{abstract}
Prediction of mutational status of different graded glioma is extremely crucial for its diagnosis and treatment planning. Currently FISH and the surgical biopsy techniques are the 'gold standard' in the field of diagnostics; the analyses of which helps to decide appropriate treatment regime. In this study we have proposed a novel approach that analyzed structural MRI image signature pattern for predicting 1p/19q co-deletion status non-invasively. A total of 159 patients with grade-II and grade-III glioma were included in the analysis. These patients earlier underwent biopsy; the report of which confirmed 57 cases with no $1 p / 19 q$ co-deletion and 102 cases with $1 p / 19 q$ co-deletion. Tumor tissue heterogeneity was investigated by variance of cross correlation (VoCC). The marked difference in pattern of VoCC between two classes was quantified using Lomb-Scargle (LS) periodogram. Energy and the cut-off frequency of LS power spectral density were derived and utilized as the features for classification. RUSBoost classifier was used that yield highest classification accuracy of $84 \%$ for G-II and $86.09 \%$ for G-III respectively in classifying $1 \mathrm{p} / 19 \mathrm{q}$ co-deleted and $1 \mathrm{p} / 19 \mathrm{q}$ non-deleted glioma. In clinical practice the proposed technique can be utilized as a pre-confirmatory test of glioma mutation, before wet-lab validation.
\end{abstract}

\section{Introduction}

Low graded glioma (LGG) are the group of primary brain tumor that are produced from two different types of glial cells of brain called astrocytes and oligodendrocytes and are termed as astrocytomas and oligodendrogliomas respectively ${ }^{1}$. Compared to high-grade glioma (anaplastic astrocytoma, and oligodendroglioma, glioblastoma), LGG being less aggressive than other forms of glioma; thus confirms better prognosis. It is seen that genetic risk factor plays a key role in glioma prognosis. Hence determining the mutational status is rapidly becoming the integral part of the routine pathological study of gliomas that provide both diagnostic and prognostic information. At present, the popular approaches for determining mutation are DNA sequencing, immune-histochemical staining and FISH test ${ }^{2}$. But all of these techniques are invasive in nature. Thus the objective of our study is to explore a novel approach that could able to predict mutational status in different graded glioma in a non-invasive manner. In this regard we have determined chromosomal arms 1p/19q co-deletion status in grade-II (G-II) and grade-III (G-III) glioma. Numerous imaging assays have been executed to determine the molecular characteristics and prognostic markers in LGG using multi-modal medical images ${ }^{3-6}$. However many of these studies could not able to show satisfactory results in order to determine the $1 \mathrm{p} / 19 \mathrm{q}$ co-deletion status in individual patients.

Our study aims to determine the $1 \mathrm{p} / 19 \mathrm{q}$ mutational status non-invasively by assessing the volumetric tumor heterogeneity in mutated (1p/19q co-deleted) and wildtype (1p/19q non-deleted) gliomas from multi-parametric structural MRI. In the current study we have proposed a new "Cross correlation-Periodogram Model" to determine the 1p/19q co-deletion status in LGG.

\section{Results}

The entire code was written in MATLAB, version R2018a. In case of 1p/19q co-deleted subjects the derived VoCC metric showed consistency in the VoCC pattern between the two consecutive slices, while the wild type cases represented a random pattern in VoCC (Figure 3). The high variation and disparity in VoCC pattern for wildtype glioma indicates the change in heterogeneity across glioma slices. whereas the similarity with relatively low variation in VoCC for mutated glioma showed the homogeneity across glioma slices. This noted difference was analyzed using the periodogram spectral analysis (Figure 4) where the energy and the cut-off frequency of LSPSD estimate were used as features for classification. For 1p/19q co-deleted glioma cases the observed similarity in LSPSD reveals the presence of periodic pattern in volumetric glioma slices. However for 


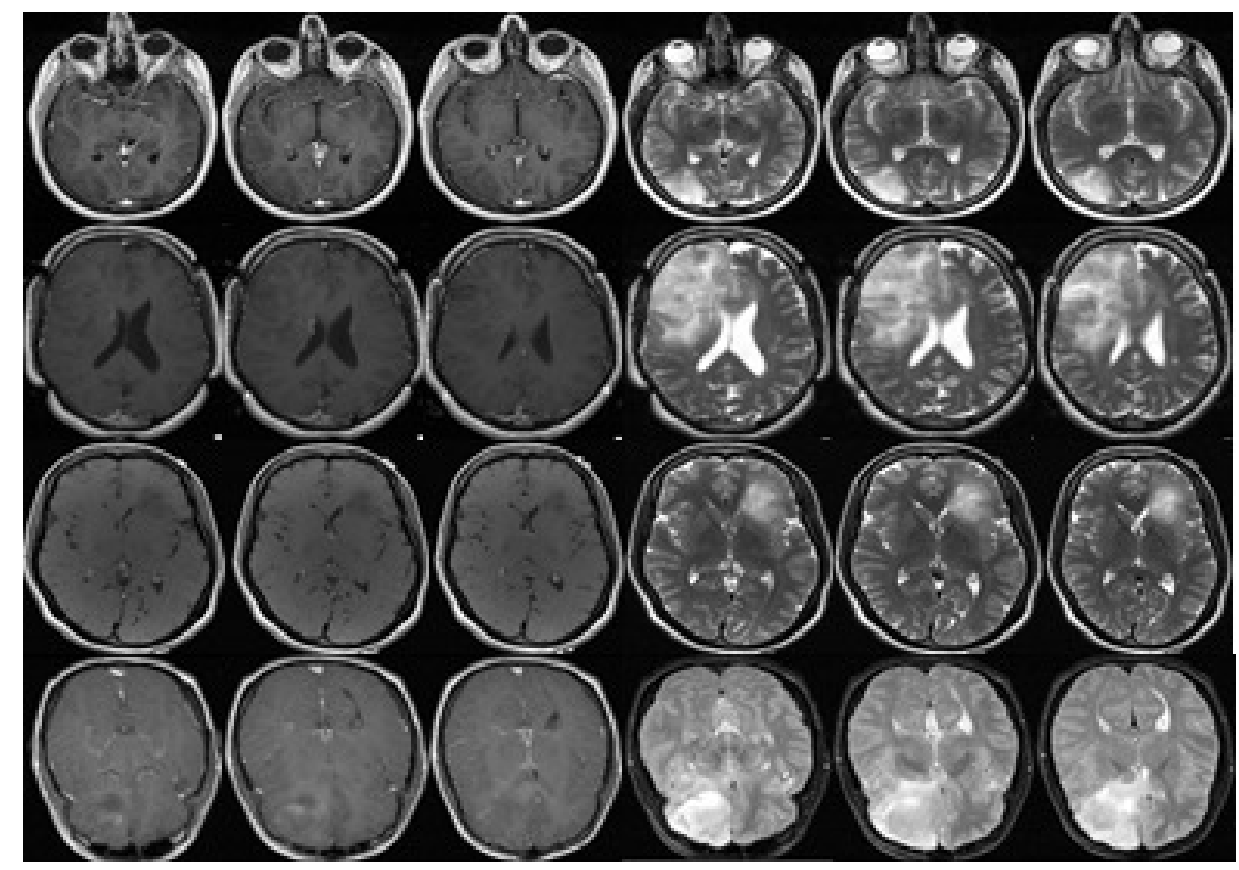

Figure 1. Representative MR Images for four subjects are collected from TCIA database. Column-1 to column-3 are post contrast T1-W MRI; column-4 to column-6 are T2-W MRI. Row-1 and row-2 represent 1p/19q co-deleted G-II and G-III glioma respectively. Row-3 and row-4 represent 1p/19q non-deleted G-II and G-III glioma respectively

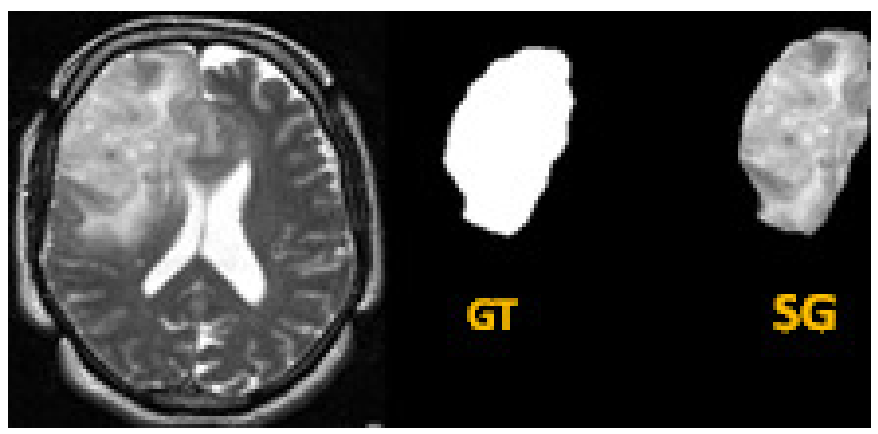

Figure 2. Glioma extraction from MRI using the ground truth. Here the abbreviations GT and SG represent the ground truth and segmented glioma respectively

1p/19q non-co-deleted cases we have found the absence in periodic patterns across MR slices. Thus, unlike 1p/19q co-deleted glioma subjects in case of $1 \mathrm{p} / 19 \mathrm{q}$ non co-deleted glioma subjects the inner product of two LSPSD estimate could not show any dominant periodic component in the glioma volume. This characteristic, seen in wildtype cases, in turn lower the cut-off frequency values and maximize the difference in energy across consecutive MR slices for wildtype glioma cases. It has to be noted that the first peak of periodogram that was observed in both classes was appeared due to the in general low frequency pattern of MRI. Hence the first peak was excluded from the analysis.

We trained the dataset with different classification algorithms including classical machine learning models- for example, support vector machine (SVM), Naive Bayes, logistic regression, as well as ensemble methods like LogitBoost and RUSBoost (Figure 5). In our study the data imbalance problem was handled using RUSBoost classifier ${ }^{7}$ where the average training accuracy of $99 \%$ was obtained. The training accuracy using SVM, Naive Bayes, Logistic regression, RUSBoost and Logit-Boost is given in Table 1. K-fold cross validation $(\mathrm{k}=10)$ was performed in our study. The obtained precision, recall, F-score and accuracy using RUSBoost classifier were reported in Table 2. Without any kind of data augmentation, our result yield better performance as compared to the result reported in literature by Z. Akkus et.al that utilized the similar TCIA dataset for the analysis ${ }^{8}$. 

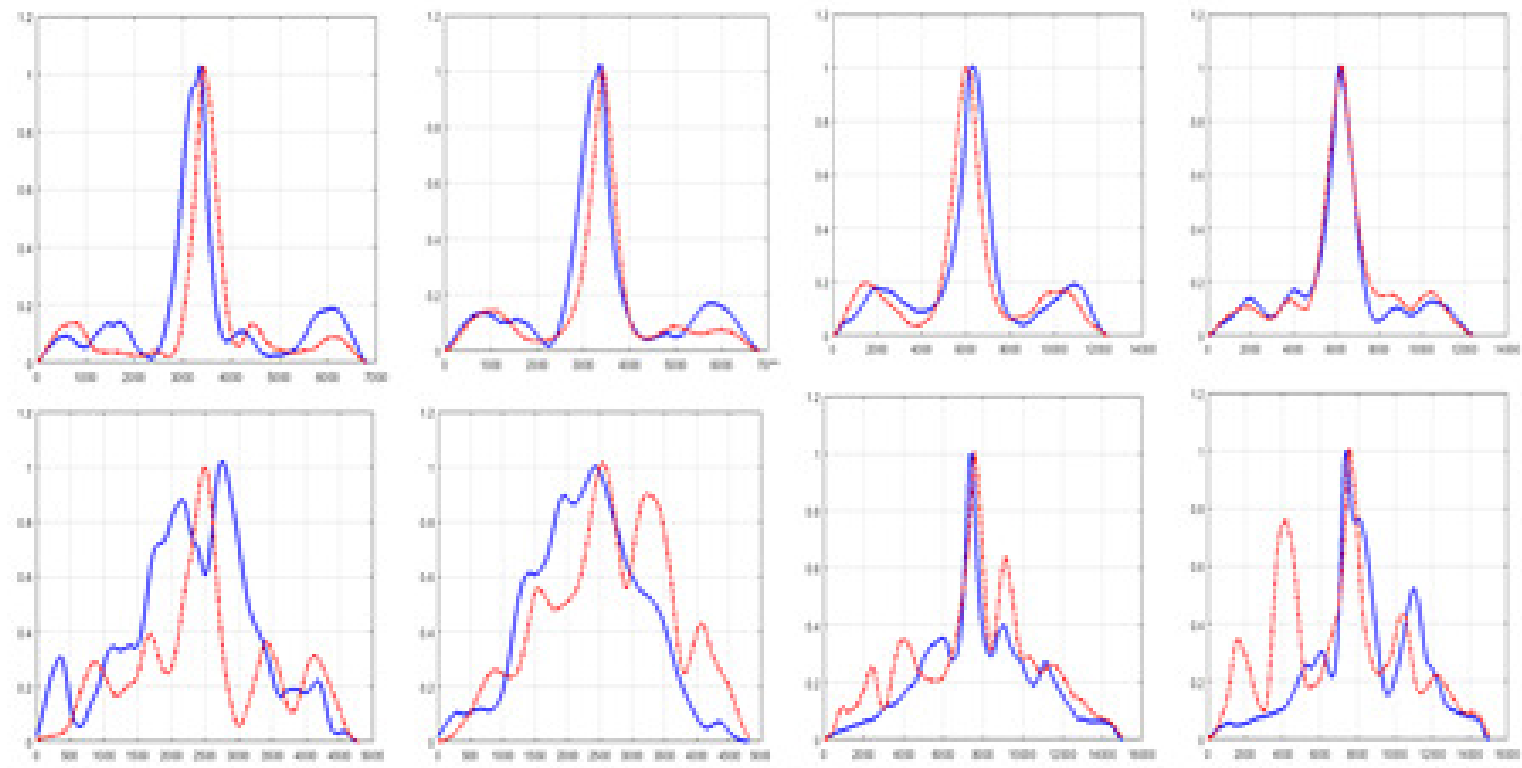

Figure 3. Plot of change in variance of cross correlation (VoCC) curve across consecutive slices (VoCC between slice-1 and slice-2 was shown by thick blue curve, whereas VoCC between slice-2 and slice-3 was shown by dotted red curve) of LGG with 1p/19q co-deletion (Top row) and 1p/19q non-deletion (Bottom row). Column 1 and column 2 plots the same for G-II glioma with T1-W and T2-W MRI respectively and column 3 and column 4 plots the same for G-III glioma with T1-W and T2-W MRI respectively. As seen from the plot, the pattern of VoCC change drastically across slices for wildtype cases whereas the pattern is similar across slices for glioma, occurred due to mutation.

\begin{tabular}{|l|l|l|}
\hline \multirow{2}{*}{ Classifiers } & \multicolumn{2}{|l|}{ Training accuracy } \\
\cline { 2 - 3 } & G-II & G-III \\
\hline Logistic regression & $58 \%$ & $66.67 \%$ \\
\hline SVM & $53 \%$ & $66.52 \%$ \\
\hline Naive Bayes & $60 \%$ & $62.87 \%$ \\
\hline Logit boost & $96.38 \%$ & $91.52 \%$ \\
\hline RUSboost & $100 \%$ & $98.5 \%$ \\
\hline
\end{tabular}

Table 1. Training accuracy using different classifiers

\section{Discussion}

In this study, we proposed a novel methodology to determine the 1p/19q co-deletion status from T1C and T2-W MRI using cross correlation-periodogram model. As it is evident that glioma that results due to mutation has better clinical outcome than those that occurred not due to mutation ${ }^{9}$, detecting the glioma mutational status is essential for the treatment prognosis. Although FISH test is the current standard for detecting chromosomal abnormalities, it suffers with some of its key limitations that prevent its application from regular use in diagnostics ${ }^{8,10}$. Alternatively, there are several imaging studies that have shown their potential to predict the same from conventional MRI and other advanced imaging modalities such as DWI, PWI, PET etc. For example in the paper of Iwadate et $\mathrm{al}^{11}$ the author concluded that $11 \mathrm{C}$-methionine PET might aid in discriminating tumors with and without $1 \mathrm{p} / 19 \mathrm{q}$ co-deletion preoperatively. Brown $\mathrm{R}$ et.al ${ }^{12}$ has shown textural measurements can be helpful in discriminating tumors with and without mutation. Fellah et $\mathrm{al}^{5}$ presented multi-parametric MRI to identify the mutational status, however the result showed no marked differences between tumors with and without $1 \mathrm{p} / 19 \mathrm{q}$ co-deletion. Jansen et al ${ }^{6}$ also derived several biomarkers using PET images to predict 1p/19q mutational status. But none of these biomarkers reliably could detect the same in individual study subjects.

Contrary to the studies reported in literature $\mathrm{s}^{3-6,8,11,12}$, we have presented a different image processing methodology that could assess the change in volumetric tumor heterogeneity by means of cross correlation-periodogram analysis in order to determine the presence of mutation non-invasively using structural MRI images. As seen from our results, our proposed method classifies T1-W 1p/19q co-deleted and non-deleted glioma with 83\% (G-III) and 82.22\% (G-II) accuracy. The classification 

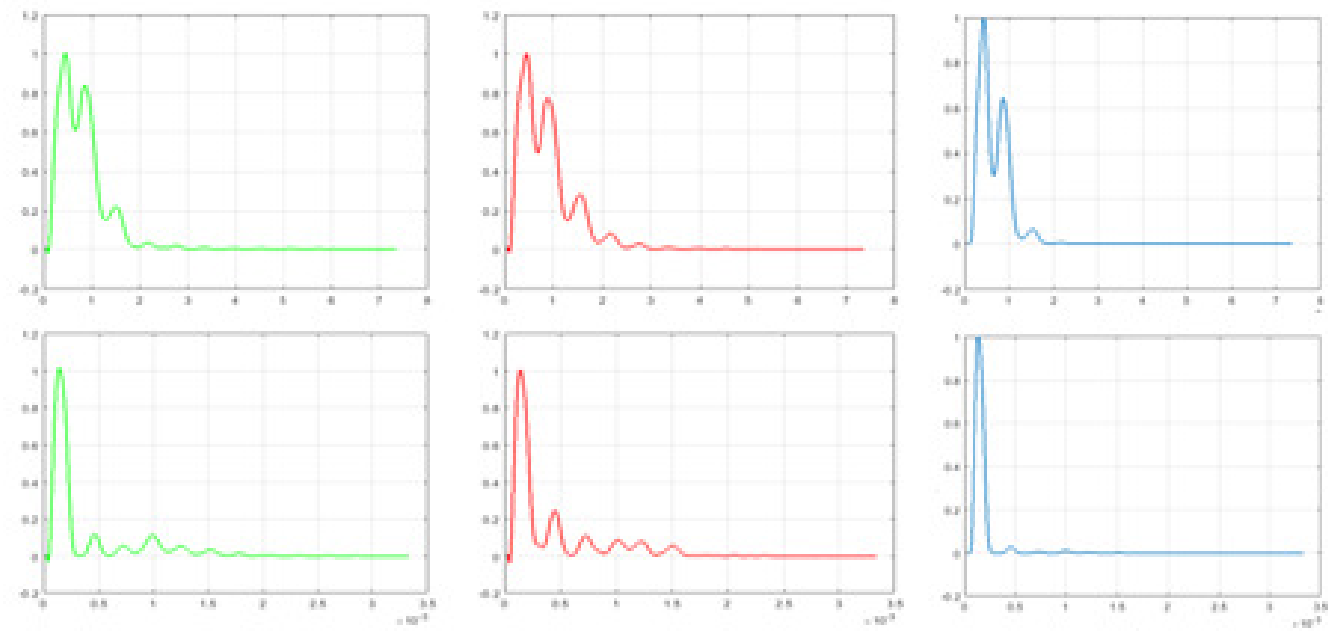

Figure 4. LSPSD estimation of VoCC for one representative glioma subject having $1 \mathrm{p} / 19 \mathrm{q}$ co-deletion (top row) and one representative glioma subject having $1 \mathrm{p} / 19 \mathrm{q}$ non-deletion (bottom row). Green curve shows the periodogram VoCC between slice-1 and slice-2. Red curve shows the periodogram VoCC between slice- 2 and slice- 3 . The inner product of two periodogram (shown in blue) showed the difference in 3D periodicity of two classes.
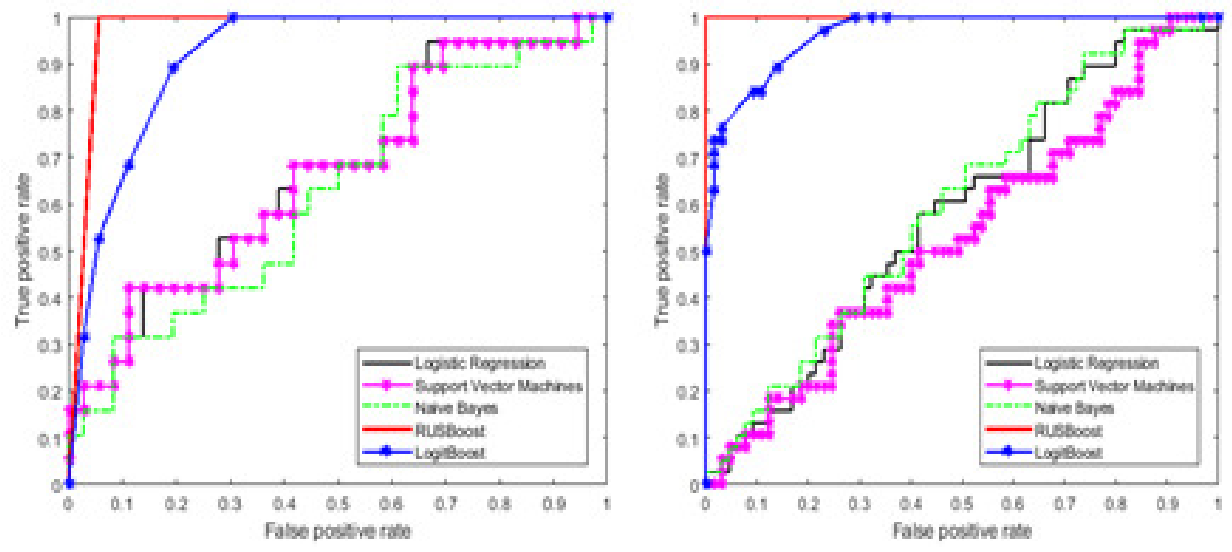

Figure 5. ROC plot using different classifiers. The best training accuracy was obtained using RUS-Boost with AUC of 0.98 (G-II) and 1 (G-III)

accuracy for G-III increases to $86.09 \%$ when using T2-W images. The misclassification rate was higher in classifying the test data with $1 \mathrm{p} / 19 \mathrm{q}$ co-deletion compared to $1 \mathrm{p} / 19 \mathrm{q}$ non-deletion. This may occur be due to the error in FISH test. As reported in literature, the reliability of FISH test is $95 \%$ and $87.5 \%$ for the predicting of $1 p$ and $19 q$ deletions, respectively ${ }^{2}$. The second reason of misclassification might be due to the skewness of the dataset. In the dataset considered here, $64.44 \%$ of the instances belong to one class (1p/19q co-deletion) that lead to lower specificity when using RUSboost classifier as it gives more weights to minority class (1p/19q non-deletion) than majority class. However while comparing with state-of-the-art studies it is seen that the current study outperforms the result reported by $\mathrm{Z}$ Akkus et. al ${ }^{8}$ that used same TCIA database and multiscale CNN approach. The classification accuracy obtained from their study was $75.6 \%$ (T2-W) and 63.3\% (T1C) using no data augmentation. The result of our proposed methodology is comparable with the result reported by Tamim Niazi et.al ${ }^{13}$, where $82.43 \%$ classification accuracy was obtianed in determining $1 \mathrm{p} / 19 \mathrm{q}$ co-deletion status. In this paper the author used radiomics features for the same glioma subjects. We also compared our approach with the study reported in ${ }^{5}$ where the authors predicted the $1 \mathrm{p} / 19 \mathrm{q}$ mutational status with $40 \%$ and $48 \%$ misclassification rates using multimodal MRI images and conventional MRI respectively. It is to be noted that the result of the current study is comparable with the result of our previously published work that utilized source distribution of VoCC to classify glioma sub-types ${ }^{14}$. Thus in clinical practice the proposed techniques can potentially be used as a pre-confirmatory test of glioma mutation before wet lab validation.

The availability of limited data size was one limitation of the current study. Hence we call for future additional investigations 


\begin{tabular}{|l|l|l|l|l|l|}
\hline Grade & LGG Status & Precision & Recall & F-score & AUC \\
\hline \multirow{2}{*}{ G-III (T1-W) } & $1 \mathrm{p} / 19 \mathrm{q}$ co-deleted & 0.85 & 0.9 & 0.89 & $87 \%$ \\
\cline { 2 - 5 } & $1 \mathrm{p} / 19 \mathrm{q}$ non-deleted & 0.90 & 0.85 & 0.83 & \\
\hline \multirow{2}{*}{ G-III (T2-W) } & $1 \mathrm{p} / 19 \mathrm{q}$ co-deleted & 0.85 & 0.9 & 0.89 & \multirow{2}{*}{$87 \%$} \\
\cline { 2 - 5 } & $1 \mathrm{p} / 19 \mathrm{q}$ non-deleted & 0.90 & 0.85 & 0.83 & \\
\hline \multirow{2}{*}{ G-II (T1-W) } & $1 \mathrm{p} / 19 \mathrm{q}$ co-deleted & 0.88 & 0.79 & 0.89 & \multirow{2}{*}{$84 \%$} \\
\cline { 2 - 5 } & $1 \mathrm{p} / 19 \mathrm{q}$ non-deleted & 0.79 & 0.88 & 0.79 & \\
\hline \multirow{2}{*}{ G-II (T2-W) } & $1 \mathrm{p} / 19 \mathrm{q}$ co-deleted & 0.83 & 0.84 & 0.87 & \multirow{2}{*}{$84 \%$} \\
\cline { 2 - 5 } & $1 \mathrm{p} / 19 \mathrm{q}$ non-deleted & 0.84 & 0.83 & 0.78 & \\
\hline
\end{tabular}

Table 2. Performance measures using RUSboost classifier on test data using LSPSD

such that the current findings can be well-validated for a large pool of patients. However the performance of the current approach on original dataset was found to be consistent with artificially augmenting data reported in the literature that utilized machine learning approach ${ }^{8}$.

\section{Methods}

\section{Subjects}

Structural MRI images of glioma are acquired from "The Cancer Imaging Archive (TCIA)" online database ${ }^{15}$, available for public access. It contains 2 different contrasts, which are $1 \mathrm{~mm}$ thick sliced T1 post contrast (T1C) and $3 \mathrm{~mm}$ thick sliced T2-weighted images of grade-2 (G-II) and grade-3 (G-III) gliomas. A total of 159 subjects were scanned, diagnosed with preoperative G-II and G-III glioma, having biopsy proven 1p/19q co-deletion and non-deletion were used in this study (Figure 1). The dataset comprises of 57 non-deleted ( $n=38$ for G-II; $n=19$ for G-III) and 102 co-deleted ( $n=65$ for G-II; $n=36$ for G-III) glioma subjects. The dataset also contains the ground truth of segmented glioma of consecutive 3 slices in each subject including the one with the biggest tumor diameter and ones just below and above it. Hence a total of 477 slices ( 3 slices per LGG subject) were incorporated in this study. All images were acquired at 1.5T or 3T on either Siemens Medical System (Malvern, PA, USA) or General Electric Medical System (Waukesha, WI, USA) scanner. Figure 1 shows one subject of LGG MRI characteristics in T2-W and T1C-W images having 1p/19q co-deletion and non-deletion.

\section{Glioma segmentation and data normalization}

The glioma portion was extracted from MRI using the ground truth provided in TCIA database. Here the ground truth images was utilized as a mask to segment the whole glioma (Figure 2). Post segmentation, each glioma image was normalized by z-scores using Equation 1 in order to balance the intensity.

$$
z=\frac{X(i, j)-\mu}{\sigma}
$$

where, $\mu$ and $\sigma$ denotes the mean and standard deviation of the image $X(i, j)$ respectively.

\section{Proposed methodology}

\section{Detection of tumor tissue heterogeneity across slices by VocC}

The change in tumor heterogeneity across slices was investigated by means of cross correlation (CC) that evaluate whether two successive slices, (here, glioma ROI) of MRI image volume exhibit common features, and therefore are correlated. Hence CC analysis is likely to reveal if the differences in molecular characteristics of glioma lead to differences in structural layout across slices.

Given two successive glioma slices $\mathrm{X}$ and $\mathrm{Y}$, the 2D cross correlation function is defined as

$$
C(i, j)=\sum_{m=0}^{M-1} \sum_{n=0}^{N-1} X(m, n) Y(m-i, n-j)
$$

where, $-(M-1) \leq i \leq(M-1)$ and $-(N-1) \leq j \leq(N-1)$

In order to assess the change in tumor volume heterogeneity between two successive slices, we have proposed a new function "Variance of $C C^{\prime \prime}($ VoCC) that examines the change in CC for different values of lag. The VoCC was derived as:

$$
\sigma_{C C}^{2}=\frac{\sum_{i=1}^{2 M-1}(C(i, j)-\bar{C}(\bullet, j))^{2}}{2 M-2}
$$


where,

$$
\bar{C}(\bullet, j)=\frac{1}{2 M-1} \sum_{i=1}^{2 M-1} C(i, j)
$$

The VoCC of 3 successive glioma slices at different lags were plotted for the two classes having (i) 1p/19q co-deletion and (ii) $1 \mathrm{p} / 19 \mathrm{q}$ non-deletion and this is shown in Figure 3. Since VoCC quantifies the change in uniformity of the intensity values across successive slices, this measure is relevant in examining the volumetric behaviour (across-slice behaviour) between mutant and wildtype glioma. One application of the proposed VoCC was reported in our previous publication ${ }^{14}$ where the source distribution of VoCC was computed in order to check if the significant differences exist between two glioma classes.

\section{Feature Extraction: Examining the presence of 3D periodicity in 1p/19q co-deleted and non-deleted glioma}

The VoCC plots for the two classes show marked visible differences (Figure 3). The essence of these visible differences are captured by extracting suitable features that would be useful for classification between mutant and wildtype glioma. As discussed before, in our prior work the source distribution of VoCC showed significant differences between two glioma sub-types. In the current study the applicability of VoCC to assess glioma heterogeneity is further investigated by determining its volumetric periodicity. Hence the power spectral density (PSD) estimate of the VoCC corresponding the two classes was computed using Lomb-Scargle power spectral density (LSPSD) estimate in order to illustrate the differences in spectral signature of two classes. Lomb (1976) and Scargle (1982) postulated the Lomb-Scargle periodogram; an algorithm that helps in detection and characterization of periodicity ${ }^{16,17}$. There are few works reported in literature that utilized LS periodogram in order to find periodic patterns in the field of genetics and biological rhythmic process ${ }^{18-21}$.

The LS periodogram was formulated as below:

$$
\left.P_{L S}(f)=0.5 \frac{\sum_{n} \sigma_{C C}^{2} \operatorname{Cos}\left(2 \pi f\left[t_{n}-\tau\right]\right)^{2}}{\sum_{n} \sigma_{C C}^{2} \operatorname{Cos}^{2}\left(2 \pi f\left[t_{n}-\tau\right]\right)}+0.5 \frac{\sum_{n} \sigma_{C C}^{2} \operatorname{Sin}\left(2 \pi f\left[t_{n}-\tau\right]\right)^{2}}{\sum_{n} \sigma_{C C}^{2} \operatorname{Sin}^{2}\left(2 \pi f\left[t_{n}-\tau\right]\right)}\right)
$$

where, $\sigma_{C C}^{2}$ is VoCC which is a function of ' $t$ ', given by Equation 3, $\tau$ is the time delay and is specified for each frequency 'f' to ensure time shift invariance: $\tau=\frac{1}{4 \pi f} \tan ^{-1} \frac{\sum_{n} \sin \left(4 \pi t_{n}\right)}{\sum_{n} \operatorname{Cos}\left(4 \pi t_{n}\right)}$

Volumetric 3D periodicity of LGG subject was measured by taking the inner product of periodogram of VoCC between two successive slices. If the VoCC plots of successive slices exhibit different pattern, The inner product of the respective periodogram will exhibit the following nature:

(i) The inner product of two corresponding spectrum will be significantly different compared to the input spectrum due to presence of unequal number of peaks. As a result the corresponding peaks of two VoCC may not coincide at similar location. Also there will be large difference in corresponding peak amplitude of two periodogram

(ii) This will result a nearly flat spectrum with reduced oscillation.

Conversely, dominant peak will be visible in both the spectrum at same location when VoCC across slices exhibits similar pattern. As a result, the inner product of respective periodogram will also show a similar profile as the input spectrum with comparatively more oscillations.

The above concept of determining the change in 3D periodicity across MR slices was executed for each LGG subject to predict the presence of $1 \mathrm{p} / 19 \mathrm{q}$ co-deletion. We hypothesize, the change in periodicity across slices is negligible for cases with $1 p / 19 q$ co-deletion. This change in volumetric periodicity is quantified by extracting suitable spectral features for classification. The extracted spectral features include:

(i) Difference energy between two periodograms,

(ii) Total volumetric energy: It is defined as the total energy of the inner product of two peridograms.

(iii) Cut-off frequency of 3D LSPSD: It is defined as the frequency at which the amplitude of LSPSD estimate is nearly equal to zero. The LSPSD estimate of VoCC for one glioma subject with 1p/19q co-deletion and non-deletion is shown in Figure 4.

\section{RUSBoost Classification}

The dataset considered in our study is poorly balanced with a ratio of 2:1 (mutated:wildtype). In such cases constructing an effective classification model is a challenging task. When examples of a specific class greatly outnumber the examples of another class (data imbalance), the performance of traditional machine learning classification models drop significantly. These algorithms tend to only predict the majority class (negative class) data where the minority class (positive class) are treated as noise and are often ignored. Thus, there is a high probability of misclassification of the positive class by classifying all instances as negative class. Two most commonly used techniques in order to improve this class imbalance problem are data sampling and boosting ${ }^{7}$. The class distribution is balanced by sampling technique that either removes samples from the majority class (under-sampling) or add samples to the minority class (oversampling). Alternatively, boosting is an advance data sampling 
technique that can improve the performance of any weak classification model by iteratively building an ensemble models. In each iteration step, the weights of the sample which were incorrectly classified during the current iteration are modified. Such technique is very effective when dealing with class imbalance problem where the higher weights are given to the minority class examples which are likely to be misclassified in subsequent iterations. RUSBoost is one example of hybrid sampling/boosting algorithm that incorporates random undersampling (RUS)- a technique that removes data samples randomly from the majority class $^{7}$.

Let ' $\mathrm{n}$ ' examples in dataset ' $\mathrm{V}$ ' are represented by tuple $\left(x_{k}, y_{k}\right)$ where $x_{k}$ is a point in feature space ' $\mathrm{X}$ ', and $y_{k}$ be the class label in a set of class label ' $\mathrm{Y}$ '. The algorithm begins with initializing the weight of each example to $1 / \mathrm{n}$ where 'n' is the number of training examples. If the total number of iterations are denoted by ' $\mathrm{P}$ ' (represents the number of classifiers in the ensemble model), then $\mathrm{P}$ weak hypothesis $H_{t}$ are iteratively trained ( $\mathrm{t}=1$ to $\mathrm{P}$ ) using some classification algorithm 'WeakLearn' as follows: First, RUS removes majority class examples until both the minority and majority class examples are balanced (1:1). This will result a new training dataset $V_{t}^{\prime}$ having a new weight distribution $W_{t}^{\prime}$. In the next step, $V_{t}^{\prime}$ and $W_{t}^{\prime}$ are passed to 'WeakLearn' (base learner) in order to create the weak hypothesis $H_{t}$. Based on actual training dataset ' $\mathrm{V}$ ' and weight distribution ' $W_{t}$ ', the pseudo-loss $\delta_{t}$ is calculated. After this, the distribution of weights for the next iteration $W_{t+1}$ is updated using weight update parameter $a_{t}$ followed by normalization. Finally, after ' $\mathrm{P}$ ' iterations the study hypothesis $\Theta(x)$ is returned as a weighted vote of the each weak hypothesis.

\section{Data availability}

The dataset analysed during the current study are available in The Cancer Imaging Archive (TCIA) repository. The dataset can be downloaded from this link:

https://wiki.cancerimagingarchive.net/display/Public/LGG-1p19qDeletionbef9e2ed4c354a92bae9ff35e8449e31

\section{References}

1. Kleihues P, S. B., Burger PC. The new who classification of brain tumours. Brain Pathol. 3(3), $255-268$ (1993).

2. Scheie D, A. P. Fluorescence in situ hybridization (fish) on touch preparations: a reliable method for detecting loss of heterozygosity at 1p and 19q in oligodendroglial tumors. The Am. J. Surg. Pathol. 30(7), 828-837 (2006).

3. Latysheva A, B. P., Emblem KE. Dynamic susceptibility contrast and diffusion mr imaging identify oligodendroglioma as defined by the 2016 who classification for brain tumors: histogram analysis approach. Neuroradiology (2019).

4. Kebir Sied, L. L. Hybrid 11c-met pet/mri combined with machine learning in glioma diagnosis according to the revised glioma who classification 2016. Clin. Nucl. Medicine 44(3), 214-220 (2019).

5. Fellah S, C. D. Multimodal mr imaging (diffusion, perfusion, and spectroscopy): is it possible to distinguish oligodendroglial tumor grade and $1 \mathrm{p} / 19 \mathrm{q}$ codeletion in the pretherapeutic diagnosis? Am. J. Neuroradiol. 34(7), 1326-1333 (2013).

6. Nathalie L. Jansen, V. G., Christoph Schwartz. Prediction of oligodendroglial histology and loh 1p/19q using dynamic fet-pet imaging in intracranial who grade ii and iii gliomas. Neuro Oncol. 14(12), 1473-1480 (2012).

7. Seiffert, C. \& M, T. Rusboost- a hybrid approach to alleviating class imbalance. IEEE transactions on systems, man, cybernetics 40, 1-5 (2010).

8. Z Akkus, J. S. J. P. A., Issa Ali. Predicting deletion of chromosomal arms 1p/19q in low-grade gliomas from mr images using machine intelligence. J. Digit. Imaging 30, 469-476 (2017).

9. Lei Deng, Y. L. X. B., Pengju Xiong. Association between idh1/2 mutations and brain glioma grade. Oncol. Lett. 16(4): 54055409 (2018).

10. Eastmond DA, M. \& D.S.Rupa. Advantages and limitations of using fluorescence in situ hybridization for the detection of aneuploidy in interphase human cells. Mutat. Res. 348(4), 153-162 (1995).

11. Iwadate Y, M. T. U. Y., Shinozaki N \& N, S. Molecular imaging of 1p/19q deletion in oligodendroglial tumours with 11c-methionine positron emission tomography. J. Neurol. Neurosurg. Psychiatry 87(9), 1016-1021 (2016).

12. Brown R, Z. M. The use of magnetic resonance imaging to noninvasively detect genetic signatures in oligodendroglioma. Clin. Cancer Res. 14(8), 2357-2362 (2008).

13. Saima Rathore, N. T. Imaging signature of $1 \mathrm{p} / 19 \mathrm{q}$ co-deletion status derived via machine learning in lower grade glioma. Radiomics Radiogenomics Neuro-oncology: First Int. Work. RNO-AI 2019, Held Conjunction with MICCAI-2019, LNCS 11991 61-69 (2020). 
14. Debanjali Bhattacharya, J. S., Neelam Sinha. Detection of chromosomal arms $1 \mathrm{p} / 19 \mathrm{q}$ codeletion in low graded glioma using probability distribution of mri volume heterogeneity. In Proc. IEEE region 10 conference- TENCON 2019 2695-2699 (2019).

15. Erickson, A., Bradley. https://wiki.cancerimagingarchive.net/display/public/ lgg1p19qdeletionbef9e2ed4c354a92bae9ff35e8449e31. (2017).

16. Lomb, N. R. Least-squares frequency analysis of unequally spaced data. Astrophys. Space Sci. 39(2), 447-462 (1976).

17. Scargle, J. D. Statistical aspects of spectral analysis of unevenly spaced data. Astrophys. Journal, Part 1 263, 835-853 (1982).

18. Ruf, T. The lomb-scargle periodogram in biological rhythm research: analysis of incomplete and unequally spaced time-series. Biol. Rhythm. Res. 30(2), 178-201 (1999).

19. Van Dongen, O. E. V. J., H.P \& EW, K. A procedure of multiple periods searching in unequally spaced time-series with lomb-scargle method. Biol. Rhythm. Res. 149-177 (1999).

20. Bohn, H. S. H. M. K. F., A \& U, L. Identification of rhythmic subsystems in the circadian cycle of crassulacean acid metabolism under thermoperiodic perturbations. Biol. Chem. 384, 721-728 (2003).

21. Chen J, E. G. \& AR, M. Detecting periodic pattern in unevenly spaced gene expression time series using lomb scargle periodograms. Bioinformatics 22(3), 310-316 (2006).

\section{Acknowledgements}

We would like to acknowledge the support from Visvesvaraya PhD scheme for Electronics and IT, Ministry of Electronics and Information Technology (MEITY), Government of India, for providing with the necessary fellowship to pursue research at IIIT, Bangalore.

\section{Author contributions statement}

All authors contributed to the study conception and design are contributed by all authors. Data collection and analysis were performed by D.B. Problem statement of the research was formulated by J.S. The research was performed by D.B under the guidance of N.S. The draft of the manuscript was written by D.B and N.S. All authors reviewed and approved the final manuscript.

\section{Additional information}

The author(s) declare no competing interests. 
Figures

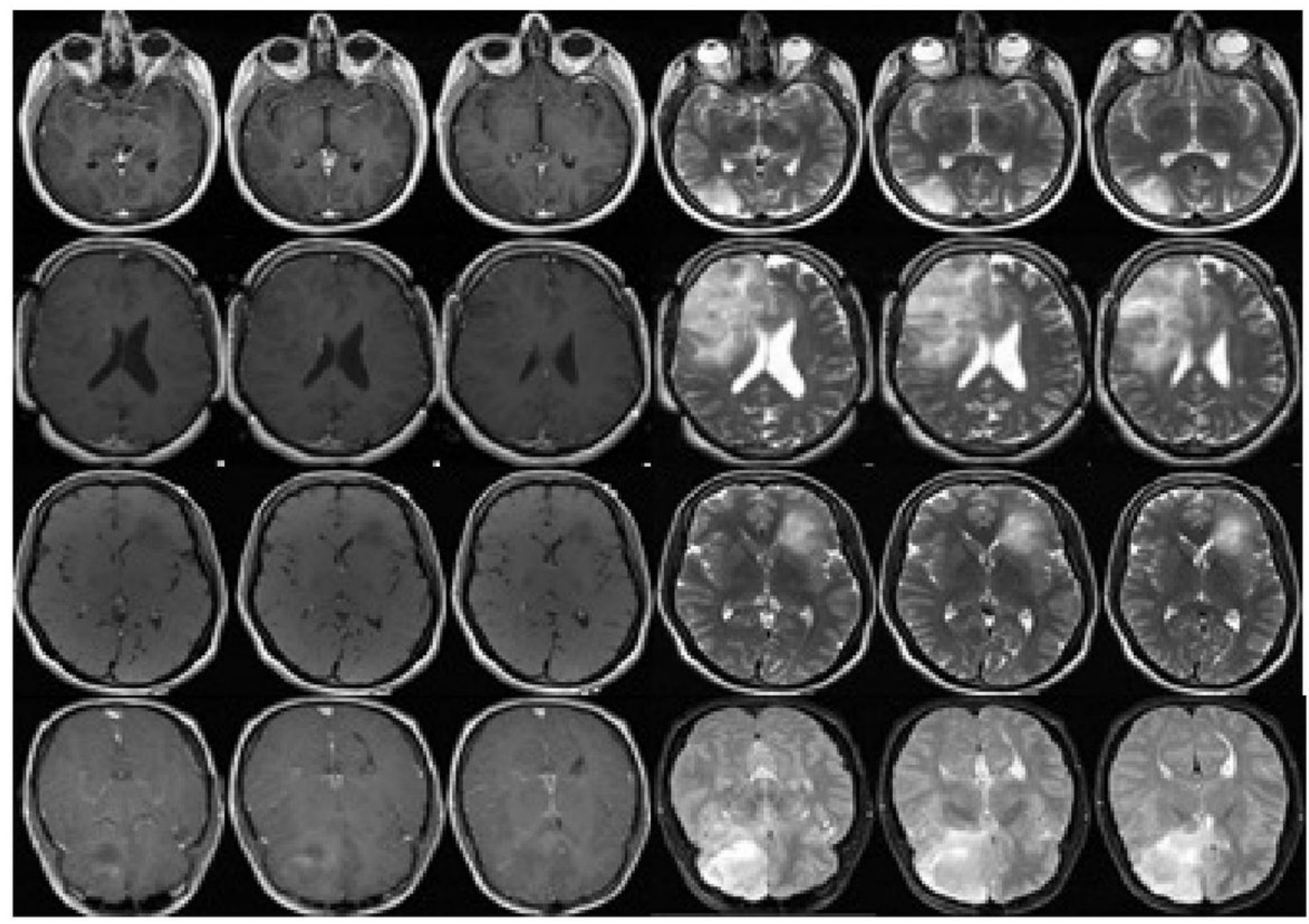

Figure 1

Please see the Manuscript PDF file for the complete figure caption 


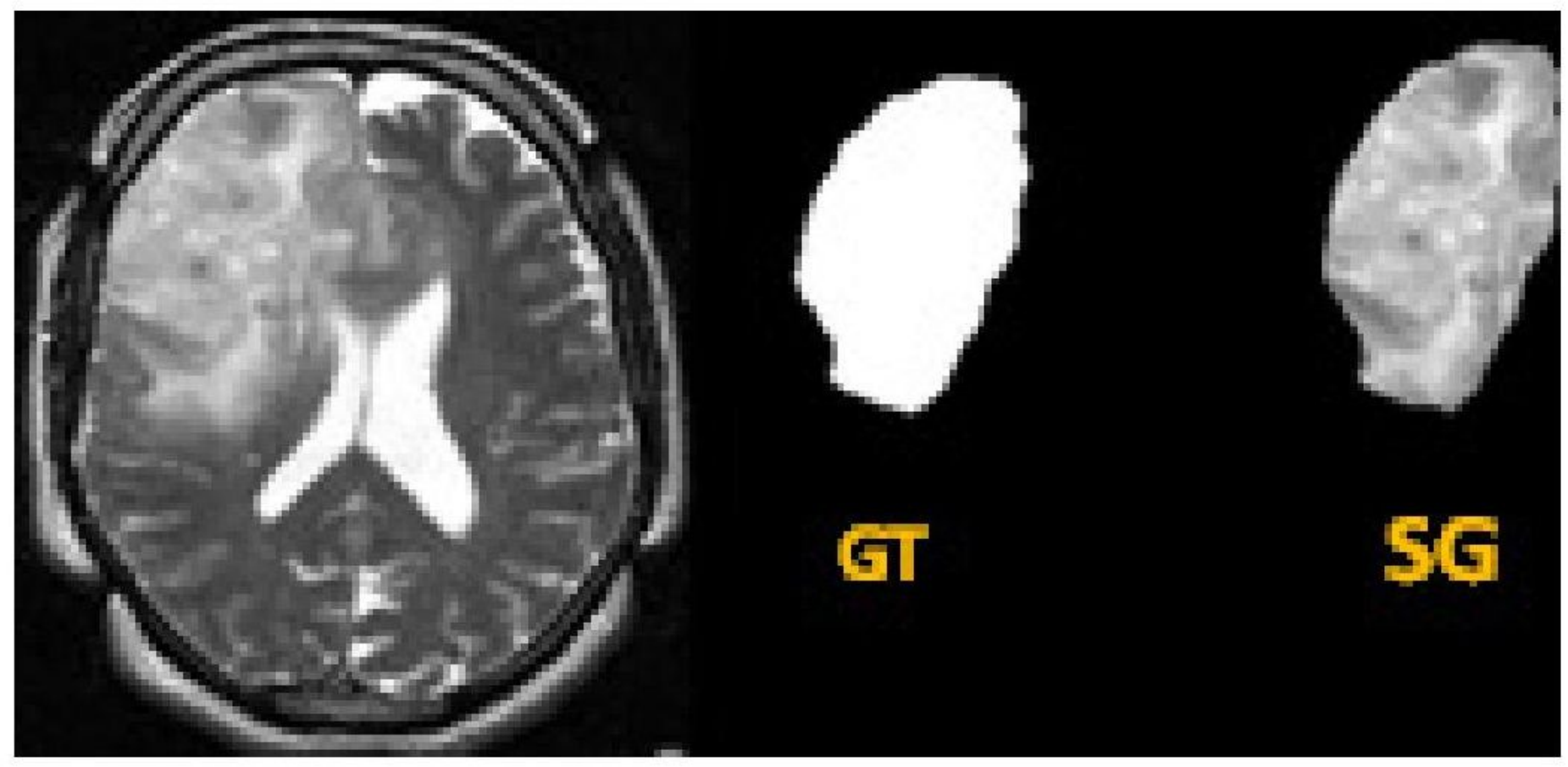

Figure 2

Please see the Manuscript PDF file for the complete figure caption
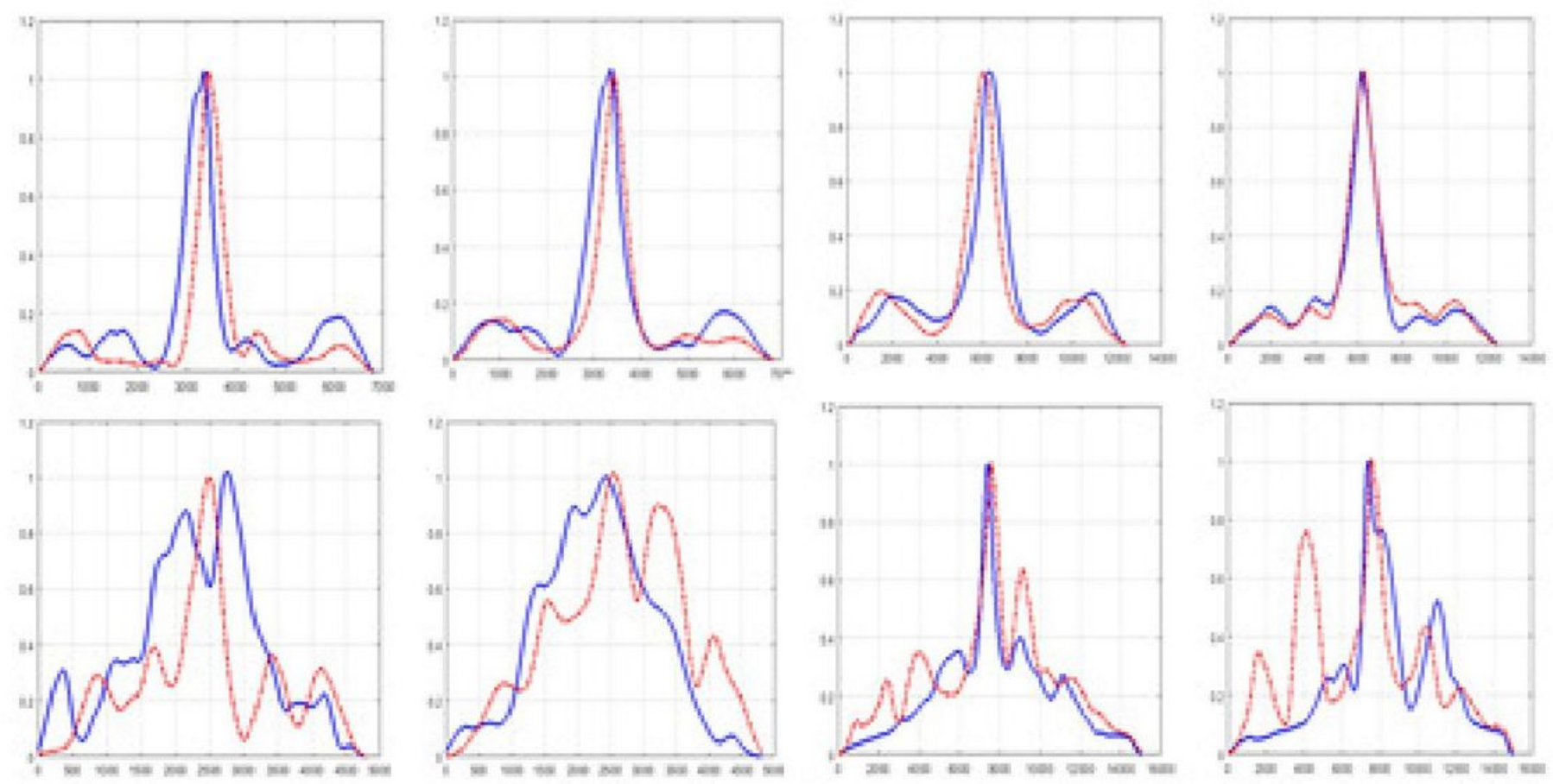

Figure 3

Please see the Manuscript PDF file for the complete figure caption 

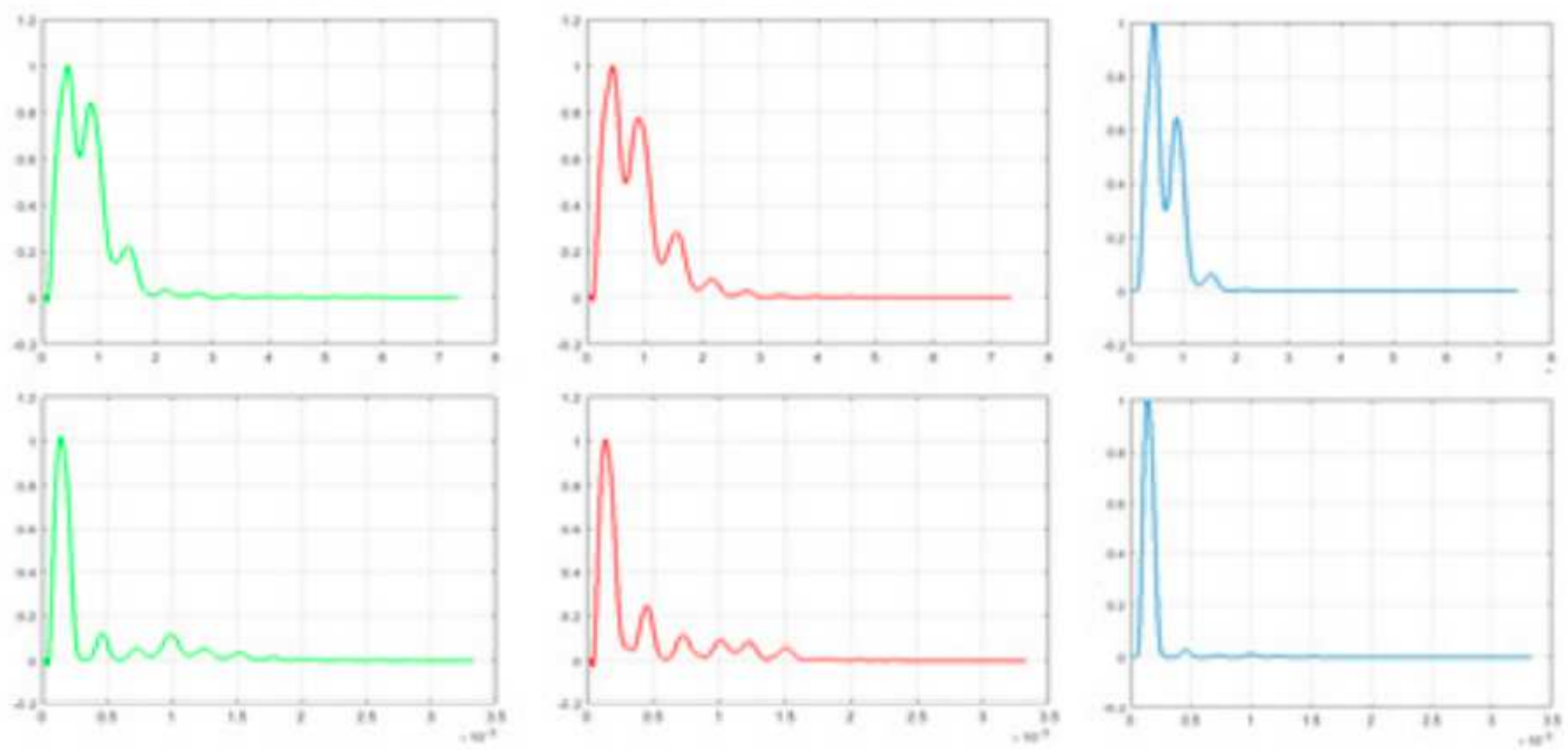

Figure 4

Please see the Manuscript PDF file for the complete figure caption
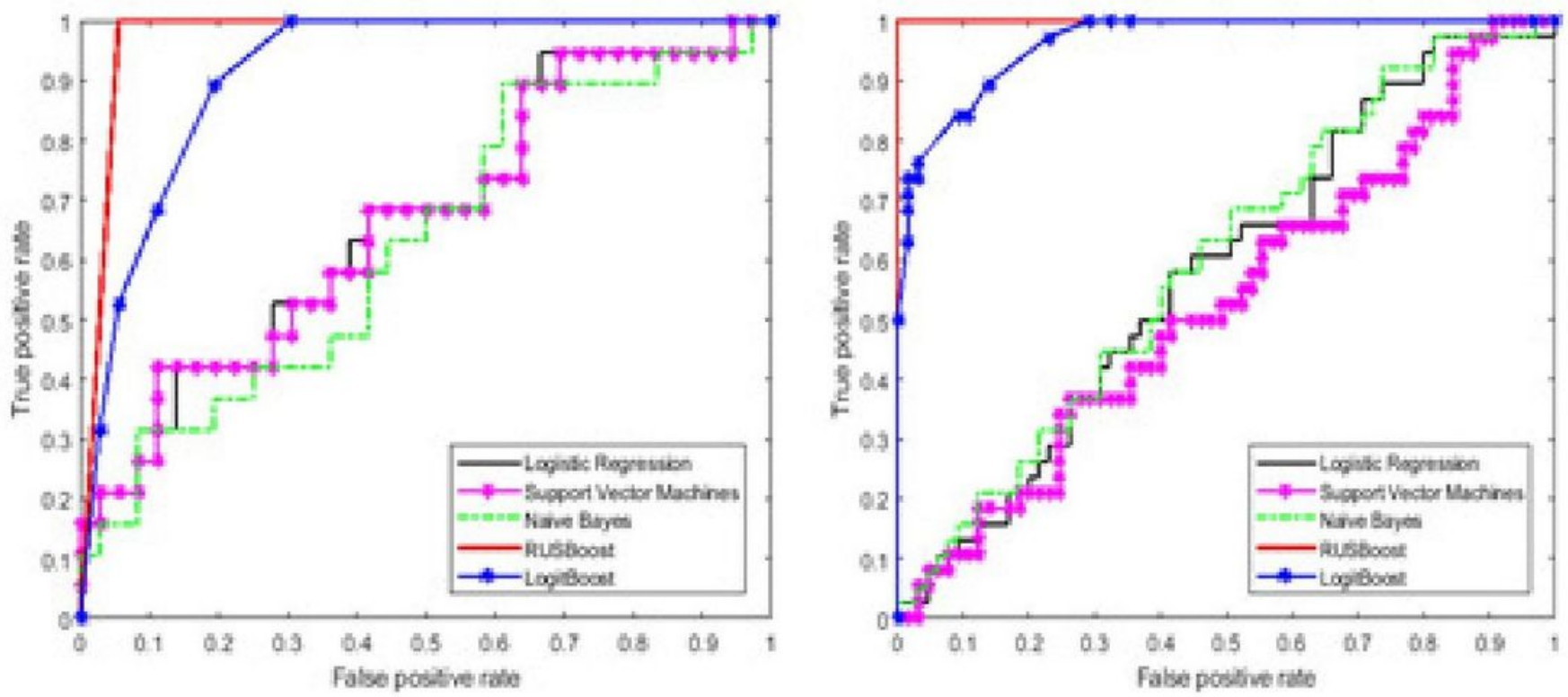

Figure 5

Please see the Manuscript PDF file for the complete figure caption 